\title{
Searching for equilibrium positions in a game of political competition with restrictions
}

\author{
Manuel Abellanas ${ }^{a}, M^{a}$ Dolores López ${ }^{b, *}$, Javier Rodrigo ${ }^{c}$ \\ a Departamento de Matemática Aplicada de la Facuitad de informática, Universidad Politécrnica de Madrid, Spain \\ - Departamento de Matemática Aplicada de la E.T.S.I. Caminos, Canales y Puertos, Universidad Politécnica de Madrid, Spain \\ 'Departamento de Matemática Aplicada, E.TS. de ingeniería. Universidad Pontificia Comillas de Madrid, Spain
}

Keywords:

A B S T R A C T

This paper considers a problem of political economy in which a Nash equilibrium study is performed in a proposed game with restrictions where the two major parties in a country vary their position within a politically flexible framework to increase their number of voters. The model as presented fits the reality of many countries. Moreover, it avoids the uniqueness of equilibrium positions. The problem is stated and solved from a geometric point of view.

$O R$ in government

Game theory

location

Political competition

Nash equilibrium

\section{Introduction}

Politics and governance consist not only in managing well, but also in generating strategies. Strategic planning of processes and political change calls for a rigorous analysis of the current situation, a clear definition of adversaries and supporters, and a precise analysis of power relations. In politics, when it comes to strategic action, tactical behavior and short-term action still prevail. For long-term conceptualization, implementing policies, and conducting campaigns, strategic planning is essential to achieve lasting success. Thus, political parties shape and vary their proposals within certain limits. These proposals are more relevant when they deal with issues in which citizens are especially interested during a certain period.

The aim of this study is to find equilibrium strategies to be followed by the two major parties of a country (government and opposition), enabling them to tune their proposals to a certain extent. Specifically, the Nash equilibrium study is proposed in a variation of the political competition game with neighborhood restrictions, as proposed by the authors in Abellanas et al. (2006). The variation proposed in this work is focused on the consideration of neighborhoods for both parties who try to win the maximum number of voters. Thus, the movements of the opposing parties, $p$ and $q$, are restricted by two separate circular neighborhoods $B=C\left(x_{0}, r\right), B^{\prime}=C\left(x_{1}, r^{\prime}\right)$, where $C(x, R)$ is the circle with center $x$ and radius $R$, which guarantee that both parties cannot adopt the same political strategies. This is important because it avoids undesirable equilibrium positions of the form $(t, t)$ and also ensures that the parties do not deviate too much from their central ideological positions $x_{0}, x_{1}$, resulting in a more "partisan" outlining of the game than the Downs game considered, for example, in Roemer (2001) and Lillo et al. (2007), where the parties are interested only in obtaining office.

The Nash equilibrium has been studied as a general model of competition. It was first stated by Nash in his dissertation Noncooperative games (Nash, 1951) as a way to obtain an optimum strategy for games with two or more players. Plott (1967), Kramer (1973), McKelvey (1976), and others have shown that pure-strategy Nash equilibria generally do not exist when competition takes place in a space of more than one dimension.

Various approaches to search for a resolution to this situation have been reported in the literature, including mixed-strategy equilibria, uncovered sets, probabilistic voting, and valence criteria. See for example (Laver and Shepsle, 1996. McKelvey, 1976, 1986, Enelow and Hinich, 1982, Lodregan and Romer, 1993, Ansolabebere and Snyder, 2000, Banks and Sundaram, 1993, and Hinich and Munger, 1995).

This work presents a discrete model of competition where examples with an infinite number of equilibrium positions are presented. The model can therefore be seen as an improvement on the classical games used in political economy. Due to the discrete nature of the presented game, the approach is developed using geometric techniques. 
A simpler version of the model has already been dealt with in previous work (Abellanas et al., 2006), but has now been adapted to reflect better the reality of many countries. Both competing parties that constitute the major parties of a country are allowed to alter their political positions on two items especially relevant for the citizens to obtain more followers. As for the choice of these two items, studying pre-election surveys can provide relevant information. To give an example, the problem of terrorism and questions related to immigration have recently become of immediate concern to the citizens of Spain. This became apparent in surveys carried out before the last elections. It should be noted that the authors do not consider that these two topics were decisive in the overall result of the elections, but that they can be important enough to deserve policy study by the parties.

Apart from these considerations, the most relevant contribution of this study as compared to previous work by the same authors is the investigation of the equilibrium in the proposed game.

The article is structured as follows:

Section 2 introduces the problem and develops the mathematical model with emphasis on its geometric analysis. In Section 3. necessary and sufficient conditions are presented for the equilibrium position to exist. Several practical examples are given which illustrate various possibilities for equilibrium.

\section{The model}

In this work, the authors generalize the study of Nash equilibrium in a political-competition game that can be interpreted as a discrete version of the Downs game (Downs, 1957; Roemer, 2001). This game is defined on a two-dimensional space where each point represents a different political position. The two players of the game represent political parties that choose their positions in the plane so as to attract the largest possible number of voters. The voters are represented by $n$ fixed points on the plane.

It is assumed here that each player captures the points that lie closer to his position than to the other player's position. The perpendicular bisector of the line joining the players' locations thus partitions the plane into two different voting regions. Each player wins the points in his own balf-plane, and the winner will be the player whose region contains more points (Serra and Revelle, 1994; Smid, 1997; Aurenhammer and Klein, 2000; Okabe et al., 2000).

The players are two parties denoted by $p$ and $q$. Their locations in the policy plane are denoted by $t_{1}, t_{2}$, determined by the policies they offer. All the political positions appearing in the voter population are represented by a finite set of types $H=\left\{p_{1}, \ldots, p_{n}\right\} \subset R^{2}$ (Roemer, 2001; Abellanas et al., 2006).

Voter preferences over the issue space are Euclidean, so the payoff functions in the game as presented are given by:

$\Pi^{1}\left(t_{1}, t_{2}\right)=$ number of points $p_{i}$ such that $d\left(p_{i}, t_{1}\right) \leqslant d\left(p_{i}, t_{2}\right)$.

$\Pi^{2}\left(t_{1}, t_{2}\right)=$ number of points $p_{i}$ such that $d\left(\mathrm{p}_{i}, t_{1}\right)>\mathrm{d}\left(\mathrm{p}_{i}, t_{2}\right)$

$$
=\mathfrak{n}-\Pi^{1}\left(t_{1}, t_{2}\right)
$$

where $d\left(t, p_{i}\right)$ is the Euclidean distance between policy $t$ and position $p_{i}$.

Both parties, $\mathfrak{p}$ and $\mathfrak{q}$, will be allowed to vary their position within a neighborhood; that is, the movements of the opposing parties with initial positions $x_{0}, x_{1}$ are restricted by two separate circular neighborlsoods, $B=B\left(x_{0}, r\right), B=B\left(x_{1}, r\right)$. One way of guaranteeing this restriction is by assigning null gains to each of them if they move outside their neighborhoods, that is, by defining $\Pi^{1}\left(t_{1}, t_{2}\right)=0$ if $t_{1} \notin B, \Pi^{2}\left(t_{1}, t_{2}\right)=0$ if $t_{2} \notin B$, with the gains as previously defined if $t_{1} \in B, t_{2} \in B$.

It should be further noted that the selection of the margins of flexibility $r, r$ is conditioned by internal characteristics of the parties. The margin of flexibility of a party basically depends on the dominant faction (reformists, opportunists, or militants) in each party. A majority of opportunists yields a larger value for $r$ because they care only about winning elections, not about ideology. However, a party with a majority of militants has less political flexibility because militants are more partisan and want policies as close as possible to the ideal policy of the party.

The following section establishes the necessary or sufficient conditions for equilibrium in the proposed model.

\section{Equilibrium with restrictions}

\subsection{Existence conditions}

Let us start by looking at a proposition that is essentially the translation of the definition of Nash equilibrium for the gains of the proposed game.

Proposition 1. The equilibrium positions in the proposed game will be the positions $\left(t_{1}, t_{2}\right)$ such that $t_{1}$ is within the area of $B$ of the maximum intersection of circles centered on a voter whose radius is the distance between the voter and $t_{2}$ (Abellanas et al., 2006) and $t_{2}$ is also within the orea of $B \prime$ of the maximum intersection.

Proof. When $q$ is located at $t_{2}$, which is within the area of maximum vote gain when $p$ is at $t_{1}$, it can be seen that if $q$ moves to $t$, then $\Pi^{2}\left(t_{1}, t\right) \leqslant \Pi^{2}\left(t_{1}, t_{2}\right)$ and the same will happen with $p$.

Proposition 2 provides a sufficient condition for the positions $\left(t_{1}, t_{2}\right)$ defined in the previous proposition to exist:

Proposition 2. Let $p_{1}, \ldots, p_{n}$ be positions in the plane of the $n$ voters. Call the points of the previous set $p_{i_{1}}^{1}, \ldots, p_{i_{k}}^{1}$, so that $d\left(p_{i_{j}}^{1}, B\right)$ $\leqslant \mathrm{d}\left(p_{i_{j}}^{1}, B^{t}\right)$. Then $p_{i_{k+1}}^{2}, \ldots, p_{i_{n}}^{2}$ are the points which make $d\left(p_{i_{j}}^{2}, B\right)>$ $\mathrm{d}\left(p_{i}^{2}, B^{t}\right)$ true, and one may write the following expression: $B_{j}^{1}=$ $C\left(p_{i}^{1}, d\left(p_{i j}^{1}, B^{i}\right)\right) \cap B, B_{j}^{2}=C\left(p_{i_{j}}^{2}, d\left(p_{i,}^{2}, B\right)\right) \cap B^{i}$.

Then, if $\bigcap_{j=1}^{k} B_{j}^{1} \neq \phi$ and $\bigcap_{j=k+1}^{n} B_{j}^{2} \neq \phi$, any position $\left(t_{1}, t_{2}\right)$ with $t_{1} \in \bigcap_{j=1}^{k} B_{j}^{1}, t_{2} \in \bigcap_{j=k+1}^{n} B_{j}^{2}$ is in equilibrium.

Proof. In the positions noted, $\mathfrak{p}$ beats the points $\mathfrak{p}_{i_{i}}^{l}, j=1, \ldots, k$ wherever $q$ is within its neighborhood, and $q$ beats the points $p_{i, j}^{2}, j=k+1, \ldots, n$ wherever $p$ is within its neighborhood. Then, if $p$ moves, it cannot gain more than the $k$ voters it has when $q$ is in position $t_{2}$, and the same situation exists for $q$; therefore, position $\left(t_{1}, t_{2}\right)$ is in equilibrium.

\section{Remarks}

1. The previous result guarantees the existence of an infinite number of equilibrium positions for the cases described. This result contrasts with the uniqueness of the equilibrium positions, when they exist, in the usual definition of games without restrictions such as Downs' game.

2. The given condition is not necessary for the existence of equilibrium; that is, there may be equilibrium positions without this condition. These possible cases are referred to in Section 3.3.

3. To verify the condition established in Proposition 2, it is possible to use the geometric construction known as an arrangement of circles (de Berg et al., 1997). This arrangement can be constructed using a randomized incremental algorithm with expected running time $O\left(n^{2}\right)$ (Edelsbrunner et al., 1992; Sharir and Agarwal, 1995). When the circles that are part of the arrangement have a non-empty intersection, this condition will hold. Otherwise, information can be obtained about the area where the maximum number of voters can be secured for each party, wherever the 
other may be, by attaching a label to each cell of the arrangement that states the number of circles containing the cell. This can be done by means of a standard sweeping algorithm (Bentley and Ottmann, 1979) with running time $\mathrm{O}\left(n^{2} \log n\right)$.

4. In the case of equidistance between the positions of both parties, the voter is assigned to the first one (see Section 2), and to guarantee that the position is in equilibrium, $t_{2}$ should be inside $\bigcap_{j=k+1}^{n} B_{j}^{2}$.

The following result presents a necessary condition regarding the gains so that a certain position may be in equilibrium. Because the gains are considered complementary, only the gain condition for the first party is considered.

Proposition 3. If $\left(t_{1}, t_{2}\right)$ is an equilibrium position, then $l \leqslant \Pi^{1}\left(t_{1}, t_{2}\right) \leqslant n-r$, where $r$ is the maximum intersection in $B$ of $C\left(p_{i}, d\left(p_{i}, B^{\prime}\right)\right)$ and $l l$ is the maximum intersection in $B$ of $C\left(p_{i}, \mathfrak{d}\left(p_{i}, B\right)\right), i=1, \ldots, n$.

Proof. Let us suppose that there is an equilibrium position $\left(t_{1}, t_{2}\right)$ with $\Pi^{1}\left(t_{1}, t_{2}\right)<l$. Then locating $p$ at a position $t$ in the maximum intersection of the sets $B_{j}^{1}$ ensures a gain $\prod^{1}\left(t, t_{2}\right) \geqslant l$. This contradicts the fact that $\left(t_{1}, t_{2}\right)$ is an equilibrium position.

If $\Pi^{1}\left(t_{1}, t_{2}\right)>n-l^{\prime}$, then $\Pi^{2}\left(t_{1}, t_{2}\right)<l^{\prime}$ (the gains are complementary); therefore, $q$ can ensure a gain of $l /$ in a position $t /$ in the maximum intersection of the sets $B_{j}^{2}$, thus improving its gain, which once more contradicts the fact that $\left(t_{1}, t_{2}\right)$ is in equilibrium.

Remark. In the case where the sufficient conditions established in Proposition 2 are fulfilled, it is possible to verify that $I+V=n$, for which in the equilibrium positions $\left(t_{1}, t_{2}\right), \Pi^{1}\left(t_{1}, t_{2}\right)=1$.Proposition 4 provides a sufficient condition for non-equilibrium, that is, another necessary equilibrium condition.

Proposition 4. If, for all $t \in B$, there exist $\left[\frac{n}{2}\right]+1$ points between $\mathfrak{p}_{1}, \ldots, p_{n}, p_{j_{1}}, \ldots, p_{j_{1\left(\frac{1}{2}+1\right.}}$, such that $\bigcap_{i=1}^{\left[p_{j}+1\right.} C\left(\mathfrak{p}_{j_{i}}, \mathfrak{d}\left(p_{j_{i}}, t\right)\right) \cap B^{\prime} \neq \phi$, and the same pattern exists for all $t \in B$, then there are no equilibrium positions in the proposed game (I 3 stands for floor).

Proof. If there were an equilibrium position $(t, t)$, then either $\Pi^{1}\left(t, t^{t}\right) \leqslant \frac{n}{2}$ or $\Pi^{2}(t, t) \leqslant \frac{n}{2}$ would hold, because the gains are complementary. If, for example, $\prod^{2}\left(t, t^{\prime}\right) \leqslant \frac{n}{2}$, then for the position $t$ of the first party in $B$, there are points $p_{j_{1}}, \ldots, p_{j_{\text {装+1 }}}$ such that $\bigcap_{i=1}^{[]_{1}+1} C\left(p_{j_{i}}, d\left(p_{j_{i}}, t\right)\right) \cap B^{\prime} \neq \phi$. Placing the second party in that intersection, there is a gain of at least $\left[\frac{\mathrm{h}}{2}\right]+1$ points $\left(p_{j_{1}}, \ldots, p_{j_{\left(\frac{\pi}{2}\right)+1}}\right)$, and therefore the gain increases, which is a contradiction because the position was in equilibrium.

This condition is not very practical because it is necessary to determine whether or not it bolds for all points in neighborhoods $B$ and $B$, and therefore it must be checked for an infinite number of points. Proposition 5 provides a more useful variation of this condition because it identifies regions of $B$ (and of $B t$ ) which cannot be part of an equilibrium position, which limits the areas which must be searched for equilibrium positions.

Proposition 5. If $t \in B$, and if there are $n-I+1$ points in the set of voters $p_{j_{1}}, \ldots, p_{j_{n-1+1}}$, such that $\bigcap_{i=1}^{n-l+1} C\left(p_{j_{j}}, d\left(p_{j_{i}}, t\right)\right) \cap b^{\prime} \neq \phi$, then no point $t_{1} \in \bigcap_{i=1}^{n-t+1} \stackrel{2}{C}\left(p_{j_{j}}, d\left(p_{j_{i}}, t\right)\right)^{r} \cap B$ can be in an equilibrium position $\left(t_{1}, t_{2}\right)$ (C is the interior of $C$ and $A^{c}$ the complementory set of $A ; l$ has the same definition as in Proposition 3).
Proof. If there is an equilibrium position $\left(t_{1}, t_{2}\right)$ with $t_{1} \in \bigcap_{i=1}^{n-1+1} \stackrel{C}{C}\left(p_{j_{i}}, d\left(p_{j_{j}}, t\right)\right)^{\mathrm{r}} \cap B$, then $\Pi^{2}\left(t_{1}, t_{2}\right) \leqslant n-l$ as a consequence of Proposition 3.

However, because $t_{1}$ is in $\bigcap_{i=1}^{n-i+1} c\left(p_{j_{1}}, d\left(p_{j_{t}}, t\right)\right)^{c}$, this leads to $d\left(p_{j_{i}}, t_{1}\right) \geqslant d\left(p_{j_{i}}, t\right)$, and therefore $C\left(p_{j_{i}}, d\left(p_{j_{i}}, t_{1}\right)\right) \supset C\left(p_{j}, d\left(p_{j_{i}}, t\right)\right)$, so $\bigcap_{i=1}^{n-i+1} C\left(p_{j_{j}}, d\left(p_{j_{i}}, t_{1}\right)\right) \cap B^{\prime} \supset \bigcap_{i=1}^{n-i+1} C\left(p_{j_{j}}, d\left(p_{j_{i}}, t\right)\right) \cap B^{\prime} \neq \phi$. Placing the second party in $\bigcap_{i=1}^{i=1+1} C\left(\mathfrak{p}_{j_{i}}, d\left(\mathfrak{p}_{j_{i}}, t_{1}\right)\right) \cap B^{\prime}$ guarantees at least $n-I+1$ voters, improving the gain, which is a contradiction because $\left(t_{1}, t_{2}\right)$ was in equilibrium.

3.2. Non-uniqueness of equilibrium positions Proposition 6 proves the non-uniqueness of equilibrium positions when they exist.

Proposition 6. If there is an equilibrium position $\left(t_{1}, t_{2}\right)$ in the proposed game, then it is not unique.

Proof. Assume that $\mathfrak{p}_{j_{1}}, \ldots, p_{j_{k}}$ are the voters of $t_{1}$ and $\mathfrak{p}_{j_{k+1}}, \ldots, \mathfrak{p}_{j_{\mathrm{tr}}}$ are the voters of $t_{2}$. Then, because $t_{2}$ is inside $C\left(p_{j_{j}}, d\left(p_{j_{j}}, t_{1}\right)\right)$ for $i=k+1, \ldots, n$, it is possible to look for a position $t /$ for the second party in $B$ t which is close enough to $t_{2}$ for $t$ to be inside $C\left(p_{j,}, d\left(p_{i}, t_{1}\right)\right)$ for $i=k+1, \ldots, n$ and for $t_{1}$ to be inside $C\left(\mathfrak{p}_{j_{1}}, d\left(\mathrm{p}_{j_{3}}, t^{\prime}\right)\right)$ for $i=1, \ldots, k$, where this is the area in $B$ of maximum gain when the second party is in $t$.

It then holds that $\left(t_{1}, t\right)$ is also an equilibrium position by Proposition 1.

\subsection{Examples}

The model proposed here has the advantage that, as noted in Remark 1 of Proposition 2, there may be voter positions where there are an infinite number of equilibrium situations for the parties. This enhances the possibilities of variation for the political positions of the rivals. Nevertheless, in this section, it will be seen that there are also examples with no equilibrium positions or with

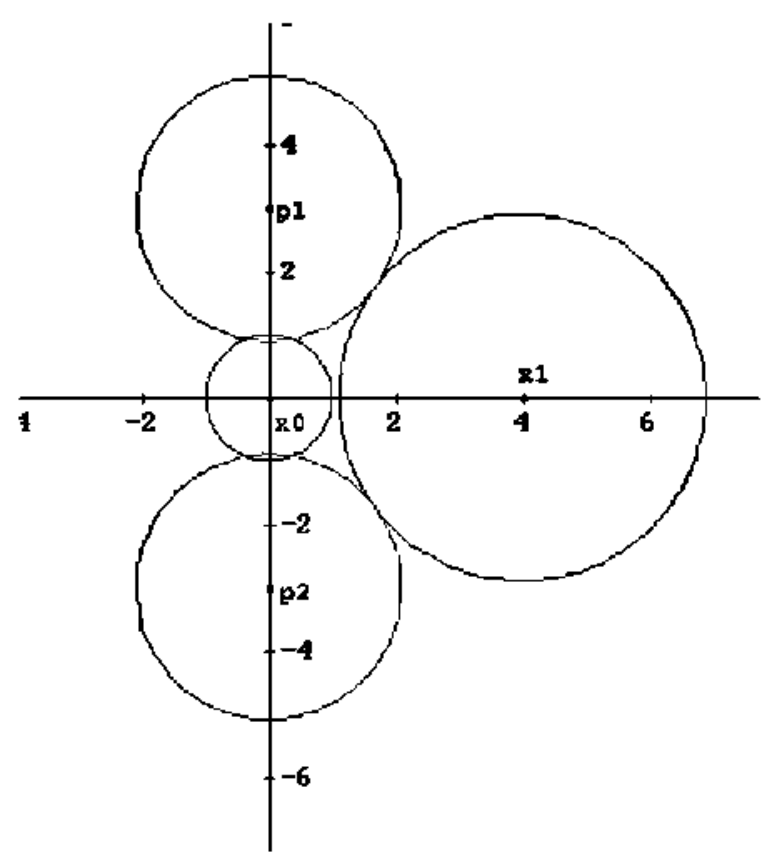

Fig. 1. Example of a situation with no equilibrium position. 
a unjque equilibrium for one of the parties, a common characteristic of political competition games without restrictions.

Example 1. Examining the situation with the following initial positions of the political parties $x_{0}, x_{1}$ and the two voters $p_{1}, p_{2}: x_{0}=(0,0), \quad x_{1}=(4,0), \quad p_{1}=(0,3), \quad p_{2}=(0,-3), \quad r=1$, $r^{\prime}=\frac{29}{10}$, it holds that $\mathfrak{d}\left(p_{1}, B\right)=\mathfrak{d}\left(p_{2}, B\right)=2<\mathfrak{d}\left(p_{1}, B^{\prime}\right)=\mathfrak{d}\left(p_{2}, B^{\prime}\right)$ $=\frac{21}{10}$ and that $B_{1}^{1} \cap B_{2}^{1}=\phi$ (see Fig. 1 ); then the condition in Proposition 2 is not fulfilled.

It can also be seen that, whenever $C\left(p_{1}, R\right)$ intersects with $C\left(\mathfrak{p}_{2}, S\right)$ for certain $R \geqslant \mathbb{d}\left(p_{1}, B\right), S \geqslant \mathbb{d}\left(p_{2}, B\right)$ (so they can intersect with $B$ ), some point of the intersection lies in $B$.

Then, for any position $t_{2}$ of the second party in $B$, it can be seen that $C\left(p_{1}, d\left(p_{1}, t_{2}\right)\right) \cap C\left(p_{2}, d\left(p_{2}, t_{2}\right)\right)$ has some point in $B$, because $R=d\left(p_{1}, t_{2}\right) \geqslant d\left(p_{1}, B^{\prime}\right), S=d\left(p_{2}, t_{2}\right) \geqslant d\left(p_{2}, B^{\prime}\right)$, and $C\left(p_{1}, d\left(p_{1}, t_{2}\right)\right)$ $\cap C\left(p_{2}, d\left(p_{2}, t_{2}\right)\right) \neq \phi\left(t_{2}\right.$ is in the intersection). Then, locating the second party in some of these points of $B$, the two points of the set will be reached in that intersection, and then any position $\left(t_{1}, t_{2}\right)$ with $\prod^{1}\left(t_{1}, t_{2}\right)<2$ is not in equilibrium. However, if $\prod^{1}\left(t_{1}, t_{2}\right)=2$, the position $\left(t_{1}, t_{2}\right)$ is not in equilibrium either, because then $\Pi^{2}\left(t_{1}, t_{2}\right)=0$. Therefore, because $C\left(p_{1}, d\left(p_{1}, t_{1}\right)\right) \cap B t \neq \phi$ or $C\left(p_{2}, d\left(p_{2}, t_{1}\right)\right) \cap B t \neq \phi$, then if the second party is in $C\left(p_{1}, d\left(p_{1}, t_{1}\right)\right) \cap B \prime$ or in $C\left(p_{2}, d\left(p_{2}, t_{1}\right)\right) \cap B$, whichever is not empty, it will win either $p_{1}$ or $p_{2}$ and improve its gain. Therefore, there is no equilibrium in this example.

Example 2. Let us consider an example that does not fulfill the condition established in Proposition 2, and in which nevertheless there are an infinite number of equilibrium positions, thus showing that the condition is not necessary:

If $x_{0}=(0,0), x_{1}=(3,0), p_{1}=(3,9), p_{2}=(3,-9), r=1, r^{r}=\frac{1}{2}$, it occurs that: $d\left(p_{1}, B\right)=d\left(p_{2}, B\right)=3 \sqrt{10}-1<d\left(p_{1}, B^{\prime}\right)=d\left(p_{2}, B^{\prime}\right)$ $=\frac{17}{2}$ and that $B_{1}^{1} \cap B_{2}^{1}=\phi$ (see Fig. 2), and then the condition of Proposition 2 does not hold.

If the second party is on $x_{1}$, and the first one in, for example, $B_{1}^{1}$ (see Fig. 3), then the first party certainly has $p_{1}$, and it cannot have

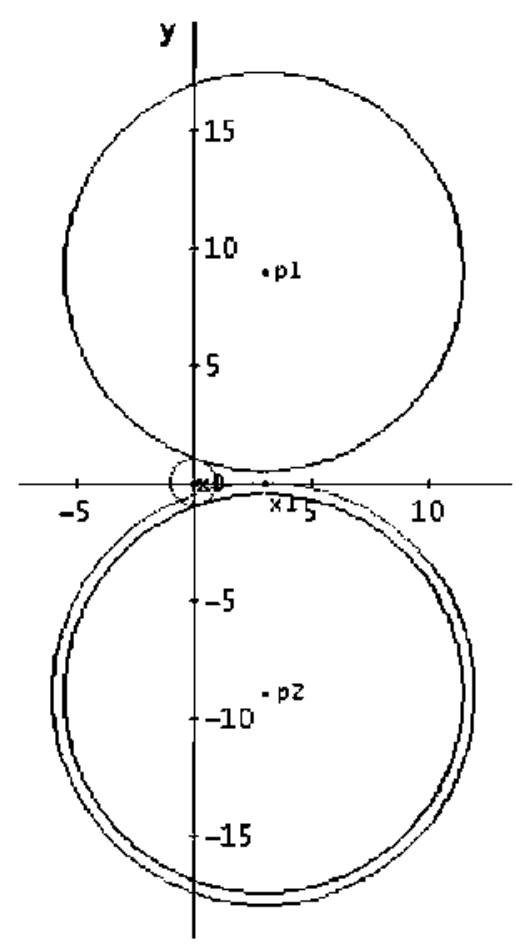

Fig. 2. Example of infinite equilibrium positions which do not comply with a sufficient condition.

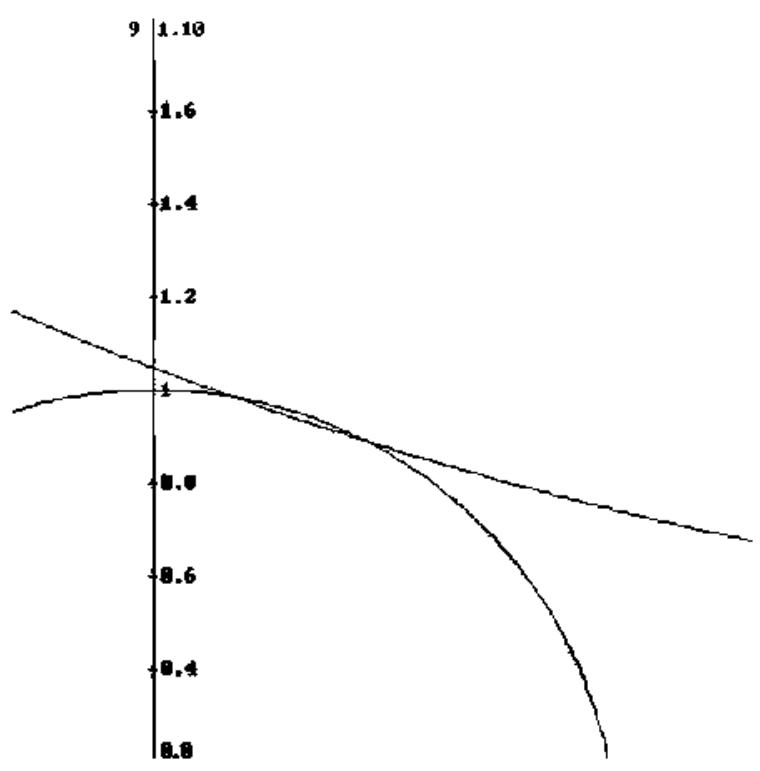

Fig. 3. Detail of the area $B_{1}^{1}$ where the first party should be located.

both by moving because $C\left(p_{1}, 9\right) \cap C\left(p_{2}, 9\right)=\left\{x_{1}\right\}$ and $x_{1} \notin B$. Then, in these positions, each party has a voter ( $p_{2}$ is the payoff for the second party, with the first one being in $B_{1}^{1}$ : see Fig. 2 ), and neither party can improve its situation by moving, so the positions considered are equilibrium positions.

Remark. In general, for any $n \geqslant 2$, there are examples of positions of $n$ points where there is no equilibrium, or where there is equilibrium without the sufficient condition being fulfilled. It is sufficient to take $n-2$ points whose maximum distance to $B$ is less than the minimum distance to $B$, such that $B_{i}^{1}=B$ for all those points and the other two points in the positions of the two previous examples (using the flexible neighborhoods for the two parties as defined in these examples).

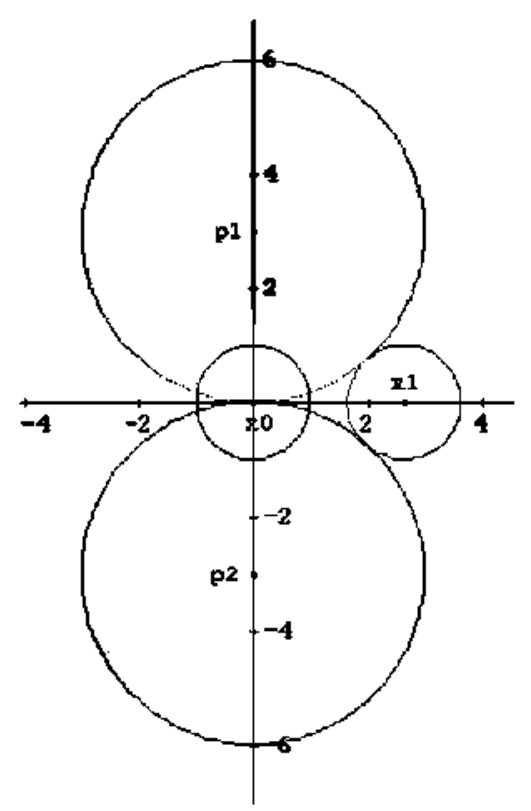

Fig. 4. Example of a unique position (not on the boundary of the neighborhood) for one of the two parties. 
Example 3. Let us consider an example where there is a unique equilibrium position for the first party and where there is no equilibrium position in which both parties are at the boundary of their neiglsborhoods. This contrasts with the result of Abellanas et al. (2006). who showed that, for a fixed position of one of the parties. there is always a position for the other that maximizes its gain at the boundary limit of its neighborhood. If we take $x_{0}=(0,0)$, $x_{1}=(\sqrt{7}, 0), p_{1}=(0,3), p_{2}=(0,-3), r=r=1$, it can be seen that $d\left(p_{1}, B\right)=d\left(p_{2}, B\right)=2<d\left(p_{1}, B^{\prime}\right)=d\left(p_{2}, B^{\prime}\right)=3 \quad$ and $\quad B_{1}^{1} \cap B_{2}^{1}=$ $\left\{x_{0}\right\} \neq \phi$ (see Fig. 4). In this example, the only equilibrium positions are $\left(x_{0}, t^{\prime}\right)$ with $t \prime \in B$, because they satisfy the condition in Proposition 2. Given that in any position $\left(t_{1}, t_{2}\right)$ with $t_{1} \neq x_{0}$ and with $\prod^{1}\left(t_{1}, t_{2}\right)=2$, it will hold that, for example, $d\left(p_{1}, t_{1}\right)>3$, after locating the second party in $B\left(p_{1}, d\left(p_{1}, t_{1}\right)\right) \cap B t^{\prime}$, it will win $p_{1}$ and improve its gain. Therefore $\left(t_{1}, t_{2}\right)$ is not an equilibrium position.

\section{Conclusions}

There are a number of localization studies in the framework of the public economy that have addressed various problems using discrete or continuous approaches (Eisets et al., 1993; Ghosh and Harche, 1993; Hakimi, 1986, 1990; Mehrez and Stulman, 1982; Church, 1984). In the equilibrium analysis of most competitive multidimensional games, it has been found that equilibrium posjtions do not exist except for singular cases, so there exist no positions for the players that guarantee that the competitor cannot increase his gain by moving.

In this paper, a discrete two-dimensional political competition model has been proposed and addressed using geometric strategies that find the equilibrium positions if they exist. The most relevant new aspect of the presented model is that it reflects the political reality of many countries, permitting the positions of the two major parties on certain topics of concern to vary to a certain extent to obtain a larger number of followers. This situation is common in regimes where the positions of the parties are not inflexible and they adapt to the preferences of the voters. The parties are in effect looking for equilibrium positions within their flexible neighborhoods. As established in Section 3, there are cases where the equilibrium positions that exist are not unique, but are regions in the plane, that is, there are an infinite number of positions. This provides more possibilities for the game and contrasts with the results for unique equilibrium positions where both parties should adopt the same position.

Although this research deals with a simplified model, this simplification applies nowadays to a large number of countries where there are two major parties.

The study of the existence and locations of equilibrium positions has been here given a wider scope by applying techniques from computational geometry, because of the discrete nature of the game as presented.

\section{References}

Abellanas, M., Lillo, l., López, M., Rodrigo. J., 2006. Electoral strategies in a dynamical democratic system: Geometric models. European Journal of Operational Research 175, 870-878.

Ansolabehere. 5., 5nyder. J., 2000. Valence politics and equilibrium in spatial election models. Public Choice 103, 327-336.

Aurenhammer, R., Klein, R., 2000. Voronoi diagrams. In: Sack, J.-R., Urnutia, J. (Eds.) Handbook of Computational Geometry. Elsevier. Ansterdam.

Banks, J., Sundaram, R.K., 1993. Moral hazard and adverse selection in a model of repeated elections. In: Barnett, W.A., Hinich, M.J., 5choefield, N.J. (Eds.). Political Econony: Institutions, Competition and Representation. Cambridge University Press, Cambridge.

Bentley. J.L., Ottmann, T.A., 1979. Algorithms for reporting and counting geometric intersections. IEEE Transactions on Computers C-28, 643-647.

Church, R., 1984. The planar maximal covering location problem. Journal of Regional Science 24 (2), 185-201.

de Berg. M., van Kreveld, M., Overmars, M., Schwarzkopf, 0. 1997. Computational Geometry. Algorithms and Applications. Springer, New York.

Downs, A., 1957. An Economic Theory of Democracy. Harper and Row.

Edelsbrunner, H., Guibas, L., Pach, J., Pollack, R., Seidel, R, Sharir, M., 1992. Arrangements of curves in the plane-topology, combinatorics, and algorithms. Theoretical Computer Science 92, 319-336.

Eisets, H.A., Laporte, G., Thisse, J.H., 1993. Competitive location models: A framework and bibliography. Transportation Science 27, 44-54.

Enelow, J., Hinich, M., 1982. Nonspatial candidate characteristics and electoral competition. Journal of Politics 44, 115-130.

Ghosh, A, Harche, F. 1993. Location-allocation models in the private sector: Progress, problems and prospects. Location Science 1, 81-106.

Hakimi, 5.l. 1986. P-median theorems for competitive location. Annals of Operations Research 5, 79-88.

Hakimi, S.L., 1990. locations with spatial interaction: Competitive locations and games. In: Mirchandani, P.B., Francis, R.L. (Eds.), Discrete Location Theory. Wiley, New York.

Hinich, M.H., Munger, M.G., 1995. Jdeology and the Theory of Political Choice. University of Michigan Press. Ann Arbor.

Kramer, G.H., 1973. On a class of equilibrium conditions for majority rule. Econometrica 42, 285-297.

Laver, M., Shepsle, K.A, 1996. Making and Breaking Governments. Cambridge University Press, Cambridge.

Lillo. l. lópez, M.. Rodrigo, J., 2007. A geometric study of the Nash equilibrium in a weighted case. Applied Mathematical Sciences 1 (55), 2715-2725.

lodregan, J., Romer. T., 1993. Polarization, incumbency, and the personal vote. In: Bamett, W.A. Hinich, M.J., Schoefield, N.J. (Eds.), Political Economy: Institutions, Competition and Representation. Cambridge University Press, Cambridge.

McKelvey, R.D., 1986. Covering, dominance, and the institution-free properties of social choice. American Journal of Political Science 30, 283-314.

McKelvin, R.D., 1976. Intransitivities in multidimensional voting models and implications for agenda control. Journal of Economic Theory 12, 472-482.

Mehrez, A., Stulman, A., 1982. The maximal covering location problem with facility placement on the entire plane. Journal of Regional Science 22 (3), 361-365.

Nash, J., 1951. Non-cooperative games. Annals of Mathematics 54, 286-295.

Okabe, A., Boots, B., Sugihara, K., Chiu, S., 2000. Spatial Tessellations: Concepts and Applications of Voronoi Diagrams. John Wiley \& Sons, Chichester.

Plott, C.R., 1967. A notion of equilibrium and its possibility under majority rule. American Economic Review 57, 787-806.

Roemer, J., 2001. Political Competition. Harvard University Press.

Serra, D., Revelle, C., 1994. Market capture by two competitors: The pre-emptive location problem. Journal of Regional Science 34 (4). 549-561.

Sharir, M., Agarwal, P., 1995. Davenport-Schinzel sequences and their geometric applications. Cambridge University Press.

Smid, M., 1997. Closest-point problems in computational geonetıy. In: 5ack, J.-R., Urrutia, J. (Eds.), Handbook of Computational Geometry. Elsevier, Amsterdam. 